\title{
Investigating Isolated Neuromuscular Control Contributions to Non-Contact Anterior Cruciate Ligament Injury Risk via Computer Simulation Methods
}

\author{
Scott G. McLean \\ University of Michigan \\ Xuemei Huang \\ Cleveland Clinic Foundation \\ Antonie J. van den Bogert

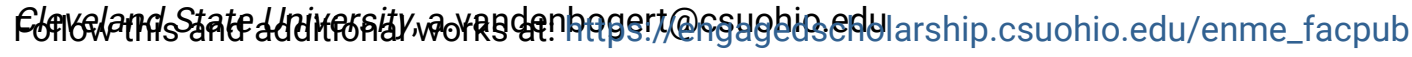 \\ Part of the Biomechanical Engineering Commons \\ How does access to this work benefit you? Let us know!
}

\section{Publisher's Statement}

NOTICE: this is the author's version of a work that was accepted for publication in Clinical Biomechanics. Changes resulting from the publishing process, such as peer review, editing, corrections, structural formatting, and other quality control mechanisms may not be reflected in this document. Changes may have been made to this work since it was submitted for publication. A definitive version was subsequently published in Clinical Biomechanics, 23, 7 , (08-01-2008); 10.1016/j.clinbiomech.2008.03.072

\section{Original Citation}

McLean, S. G., Huang, X., and van den Bogert, A. J., 2008, "Investigating Isolated Neuromuscular Control Contributions to Non-Contact Anterior Cruciate Ligament Injury Risk Via Computer Simulation Methods," Clinical Biomechanics, 23(7) pp. 926-936.

This Article is brought to you for free and open access by the Mechanical Engineering Department at EngagedScholarship@CSU. It has been accepted for inclusion in Mechanical Engineering Faculty Publications by an authorized administrator of EngagedScholarship@CSU. For more information, please contact library.es@csuohio.edu. 


\title{
Investigating isolated neuromuscular control contributions to non-contact anterior cruciate ligament injury risk via computer simulation methods
}

\author{
Scott G. McLean ${ }^{\mathrm{a}, *}$, Xuemei Huang ${ }^{\mathrm{b}}$, Antonie J. van den Bogert ${ }^{\mathrm{b}, \mathrm{c}}$ \\ a Division of Kinesiology, The University of Michigan, 401 Washtenaw Avenue, Ann Arbor, MI 48109, USA \\ ${ }^{\mathrm{b}}$ Department of Biomedical Engineering, The Cleveland Clinic Foundation, Cleveland, OH, USA \\ ${ }^{\mathrm{C}}$ Department of Orthopaedic Surgery, The Cleveland Clinic Foundation, Cleveland, OH, USA
}

\section{Introduction}

The deleterious impact of non-contact anterior cruciate ligament injuries, precipitating significant short- (Griffin

\footnotetext{
" Corresponding author.

E-mail address: mcleansc@umich.edu (S.G. McLean).
}

et al., 2006) and long-term (Lohmander et al., 2004; Maletius and Messner, 1999) debilitation, remains a significant, yet largely unsolved clinical problem. This issue is further compounded by the unexplained sex-disparity in ACL injury rates, with women suffering these injuries far more frequently than men (Agel et al., 2005; Griffin et al., 2006). Considering these facts, it is thus plausible that within the coming decades a relatively large number of 
young individuals, particularly females, will undergo substantial and potentially life altering knee joint debilitation. Hence, the elucidation and subsequent counteraction of the mechanisms of non-contact ACL is crucial.

Abnormal lower limb neuromuscular control strategies elicited during the execution of dynamic sports postures is increasingly proposed to contribute directly to non-contact ACL injury risk (Griffin et al., 2006; Hewett et al., 2006a). In particular, recent studies have suggested that lower limb postures an initial ground contact, such as combined hip and knee flexion (Chappell et al., 2007; Yu et al., 2006), and hip axial rotation positions (McLean et al., 2005a; Pollard et al., 2007), promote potentially traumatic knee joint anterior shear and/or external knee valgus load states (Chappell et al., 2007; Griffin et al., 2006; McLean et al., 2005a). Data from such studies continue to govern and refine current neuromuscular training strategies aimed at targeting and subsequently preventing these high-risk control manifestations (Bahr and Krosshaug, 2005; Hewett et al., 1999; Mandelbaum et al., 2005). Despite the continued evolution of these prevention programs, however, and reported early successes (Hewett et al., 1999), non-contact ACL injury rates and the associated sex disparity have remained (Agel et al., 2005). It seems plausible therefore, that current prevention methods may either be targeting the wrong control parameters, or inducing inappropriate control modifications through the training strategy. The continued focus on multiple neuromechanical parameters, being trained through multiple modalities, also continues to render such programs temporally and labor inefficient.

Neuromuscular control parameters currently deemed high-risk in terms of ACL injury are based primarily on the outcomes of studies adopting an in vivo human experimental model (Chappell et al., 2007; McLean et al., 2007). While such studies have indeed advanced our understanding of the intricacies of the non-contact ACL injury, their inability to examine actual injury causing events renders a precise understanding of injury mechanisms and subsequent evaluation of prevention strategy efficacies virtually impossible. Further, studies prospectively identifying a link between ACL injury risk and knee neuromechanics (Hewett et al., 2005) are no closer to explaining how these postural states manifest within the injurious movement strategy, and hence, how they can be successfully countered. The recent development and validation of subjectspecific forward dynamic sports movement simulations presents an expedited means through which to validly study acute knee injuries while controlling all aspects of the neuromuscular strategy (Erdemir et al., 2007; McLean et al., 2003, 2004a). Through this approach, the relative contributions of specific control parameters to extreme knee joint load states, and the rationale for including such parameters within the injury screening and/or prevention focus can be evaluated explicitly. With this in mind therefore, the current study utilized forward dynamics modeling methods to examine the impact of key initial contact neuromuscular control parameters on resultant knee loading during the stance phase of sidestep cutting maneuvers. Specifically, the potential for perturbations in these parameters to induce or negate injury causing anterior tibial shear and/ or knee valgus loads was explored.

To achieve these aims, and based on previous experimental literature, the following hypotheses were tested explicitly:

H1. Landing with decreased hip and knee flexion increased the risk of ACL injury via an extreme anterior shear load during perturbed sidestep stance.

H2. Landing with increased hip internal rotation and/or internal rotation velocity increased the risk of ACL injury via an extreme knee valgus load during perturbed sidestep stance.

\section{Methods}

\subsection{Subjects}

Twenty subject-specific forward dynamics lower limb models of the stance phase (0-200 ms) of a sidestep cut were generated for the current study. Subject data incorporated within each model were obtained from 10 male $($ age $=20.2(1.9) \mathrm{yrs}$, height $=184.7(8.0) \mathrm{cm}$, mass $=81.9$ $(9.8) \mathrm{kg})$ and 10 female $($ age $=21.1(3.0)$ yrs, height $=176.0$ $(11.1) \mathrm{cm}$, mass $=76.1(12.4) \mathrm{kg})$ NCAA Division 1 basketball players, who were matched for experience (males $=10.2$ (5.1) yrs, females $=10.5$ (4.8) yrs). Experience level was denoted by the number of years participating in organized basketball activity. Prior to experimentation, approval for the research was gained through the Institutional Review Board of the Cleveland Clinic Foundation and written informed consent for all subjects was obtained. Subject inclusion in the study was based on a history of no operable lower limb joint injury and no significant knee joint pain over the two-year period prior to testing.

\subsection{Data collection}

Three-dimensional (3 D) kinematic data were first recorded for each subject across 10 sidestep cutting trials via six electronically shuttered high-speed video cameras at $240 \mathrm{fps}$ and Eva 6.0 tracking software (Motion Analysis Corp., Santa Rosa, CA, USA). Synchronous ground reaction force (GRF) data were also recorded at $1200 \mathrm{~Hz}$ via an AMTI force plate (Model OR6-5, Serial \#4068, Watertown, MA, USA). Approach speeds for each trial were monitored and required to fall between $4.5 \mathrm{~m} \mathrm{~s}^{-1}$ and $5.5 \mathrm{~m} \mathrm{~s}^{-1}$, reflecting speeds at which these movements are typically executed in the game situation (McLean et al., 1999). Sidestep cutting angles were required to be $35-55^{\circ}$ from the original direction of motion, again reflecting values typically demonstrated in the game situation, and adopted in our earlier research (McLean et al., 2004b, $2005 b$ ). Subjects were required to land and sidestep cut off the right leg, such that that the cutting action necessarily moved them forward and to the left of the force plate at 
the appropriate angle. All subjects were given ample time to practice prior to data collection and were all able to execute the maneuver successfully. Previous work has shown that at least for side stepping, landing mechanics do not appear to be influenced statistically by limb dominance in athletic populations (McLean et al., 1999, 2005b). Kinematic data were obtained from the $3 \mathrm{D}$ coordinates of skin-mounted markers secured to various anatomical locations (Fig. 1) (McLean et al., 2004b). A standing trial was first collected with all joints in the neutral (assumed zero rotation) position (McLean et al., 2007). The forehead, left and right anterior superior iliac spine (ASIS), medial femoral condyle and medial and lateral malleoli markers were then removed prior to the initiation of motion trials.

\subsection{Model development and validation}

Subject-specific models were developed using in house and custom (SD/FAST - Parametric Technology Corp., Needham, MA) software and subsequently validated based on previously published methods (McLean et al., 2003, 2004a). Briefly, the standing trial video data obtained for each subject were used in conjunction with Mocap Solver 6.17 software (Motion Analysis Corp., Santa Rosa, CA, USA) to first define a kinematic model, comprising five skel- etal segments (foot, talus, shank and thigh of the right (support) limb, and the pelvis) and $12^{\circ}$ of freedom (DoF). The pelvis was assigned six (three translational and three rotational) DoF relative to the global (lab) coordinate system, with the three rotational DoF (somersault, tilt, twist) defined in accordance with the rotation sequence of Yeadon (1990). The hip joint had three rotational (flexion-extension, adduction-abduction and internal-external rotation) DoF, defined about three orthogonal axes ( $\mathrm{Wu}$ et al., 2002), passing through a fixed joint center (Bell et al., 1990). Knee joint motion was described by rotation about a fixed flexion-extension axis, passing through a joint center located according to Vaughan et al. (1990). The ankle joint was modeled as a 2-DoF mechanism, allowing rotation about talocrural and subtalar joint axes (McLean et al., 2003, 2004a). The talocrural joint center was defined as the midpoint between the lateral and medial malleoli, with the plantar-dorsi flexion axis extending laterally from this point (Isman and Inman, 1969; van den Bogert et al., 1994). The subtalar joint was located $10 \mathrm{~mm}$ directly below the talocrural joint (van den Bogert et al., 1994), with its axis oriented 41 degrees up from horizontal and $23^{\circ}$ medial from the foot axis in accordance with Isman and Inman (1969). The 3D marker trajectories recorded during the ten sidestep cutting trials for each subject were subsequently
A

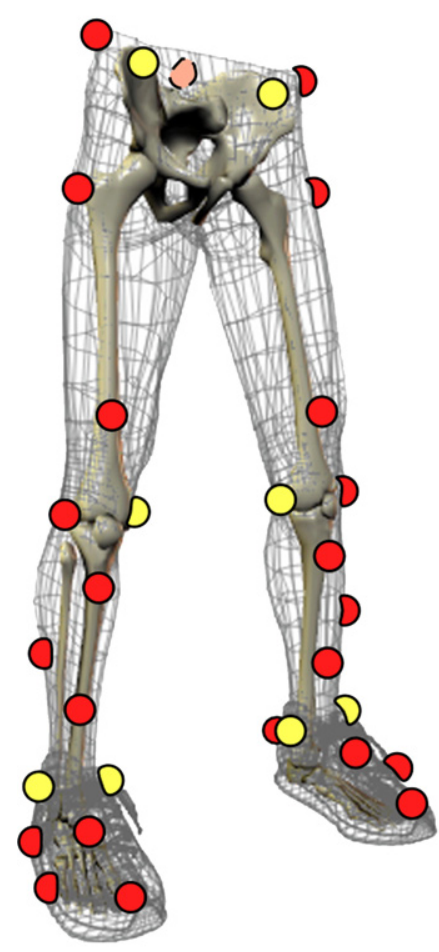

B

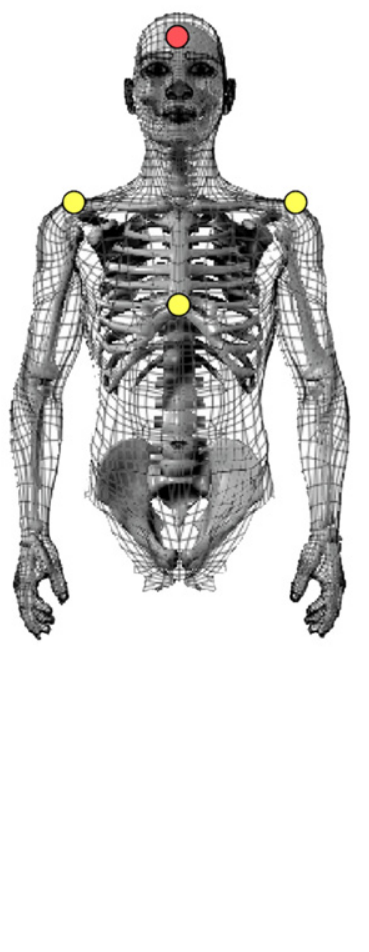

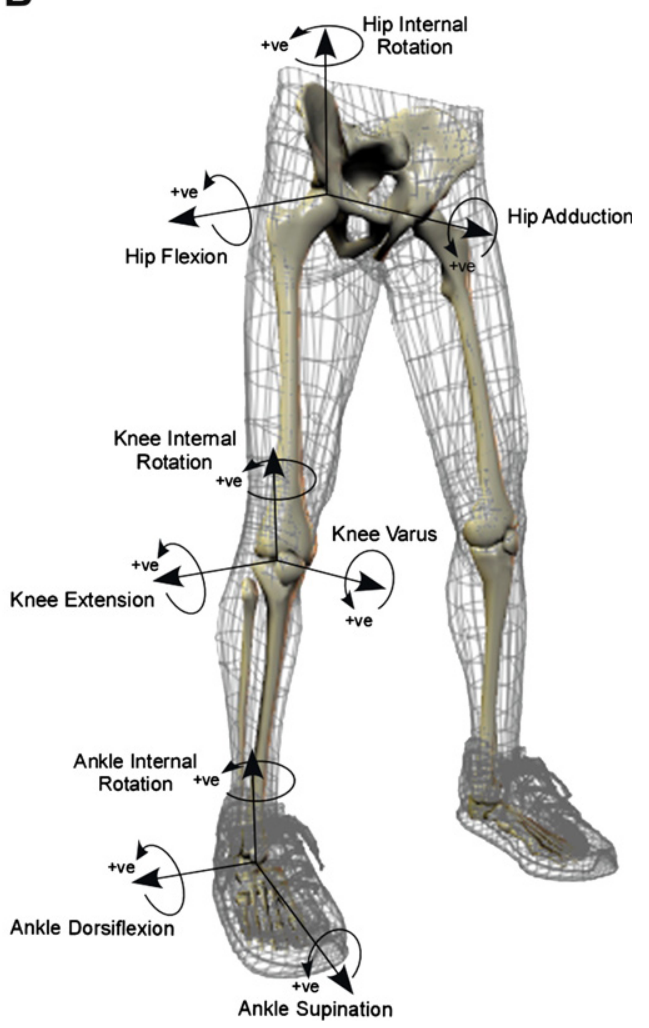

Fig. 1. Marker locations used to define a kinematic model comprised of five skeletal segments. The forehead, left and right ASIS, medial femoral condyle and lateral and medial malleoli markers (red) were removed for the recording of movement trials. For the kinematic model, Pelvis (body) motion was described with respect to the Global (lab) coordinate system via three translational and three rotational degrees of freedom. The hip, knee and ankle joints were defined locally and assigned three, one and two rotational DoF respectively. (For interpretation of the references to colour in this figure legend, the reader is referred to the web version of this article.) 
processed by the Mocap Solver software to solve for the twelve DoF of the skeleton model at each time frame $(0$ $200 \mathrm{~ms}$ ). Rotational data were subsequently normalized to each subjects' standing (neutral) pose, similar to our previous model (McLean et al., 2003, 2004a, 2004b) and in vivo experimental (McLean et al., 2007) work.

A forward dynamic 3D rigid body model of the trunk and lower extremity was generated for each subject, consisting of the skeletal model described above, with wobbling masses added to the pelvis and thigh segments, representing all body segments not modeled, including the non-support limb, arms and head (McLean et al., 2003). The wobbling mass stiffness and damping parameters were defined by discrete realistic values rather than estimating them within the ensuing optimization process. Specifically, these parameters were based on the wobbling mass being $90 \%$ of the estimated subject pelvis mass (McLean et al., 2004a, 2004b), and having an assumed natural frequency of $15 \mathrm{~Hz}$ and damping rate of $30 \mathrm{~s}^{-1}$ (Wakeling and Nigg, 2001). We have optimized these parameters in the past and found that successful model replication of a subject's sidestep movement is still possible even with erroneous and largely unrealistic stiffness and damping values (McLean et al., 2003, 2004a). Such over-fitting drastically compromises the ability of the optimized model to successfully predict of responses to random perturbations. Using fixed wobbling mass stiffness and damping values however, appears to improve both model efficacy and predictive success. Contact between the right foot segment and the ground was modeled using 91 discrete viscoelastic elements, with each element attached in precise 3D locations, which described the exterior shoe surface. The inertial characteristics of the models were based on anthropometric data obtained for each subject (de Leva, 1996) and model equations of motion were produced by SD/FAST (Parametric Technology Corp., Needham, MA, USA).

Thirty-one muscles were attached to the skeleton (Delp et al., 1990), which were categorized into 12 functionally discrete groups. A three-element Hill model was used to model muscle-tendon dynamics, with all model parameters taken from SIMM (Software for Interactive Musculoskeletal Modeling) (Musculographics, Chicago, IL, USA). The optimal length of the muscles contractile element and tendon slack lengths were also scaled to individual subject dimensions (McLean et al., 2003). Furthermore, muscle length as a function of joint angles and the associated muscle moment arms were also scale to the length of the bone closest to the muscle (McLean et al., 2004a, 2004b). For computational efficiency, the 3D muscle path models were converted into a multivariate polynomial that described musculotendon length as a function of joint angles (McLean et al., 2004a). Neural stimulation inputs for each muscle group were modeled as a piecewise linear function of time, with five parameters: the stimulation value at times 0,50 , 100, 150, and $200 \mathrm{~ms}$ after heel strike (McLean et al., 2003).

Body segment positions and velocities quantified experimentally at heel strike for each subject via the Mocap Sol- ver software, were averaged over their ten sidestep cutting trials and used as initial input conditions for the associated forward dynamic simulation. In each case, heel strike was defined as the instant when the vertical GRF first exceeded $10 \mathrm{~N}$ (McLean et al., 2005b, 2007). An ensemble average (SD) was also calculated across the ten trials from initial contact to $200 \mathrm{~ms}$ post contact for the nine rotations and three GRF's. Muscle stimulation patterns for each muscle group were subsequently optimized via a simulated annealing algorithm (Goffe et al., 1994) to minimize the difference between the model simulated and these measured subjectspecific baseline data (McLean et al., 2003). Specifically, the optimization problem was defined as

$\operatorname{minimize} J(p)=\sum_{j=1}^{12} \sum_{i=1}^{200}\left[\frac{V_{i j}-\hat{V}_{i j}{ }^{2}}{\mathrm{SD}_{j}}\right.$

where, $\mathrm{p}=\left(p_{1} \ldots p_{60}\right)$, the vector of model parameters to be optimized $V_{i j}=$ measured value (mean of all trials) of variable $j$ at time step $i \hat{V}_{i j}=$ simulation result corresponding to $V_{i j} \mathrm{SD}_{j}=$ the between-trial standard deviation in variable $j$, averaged over the 200 time samples.

Root-mean-square (RMS) fit errors and RMS prediction errors were quantified for each of the twelve baseline variables over $200 \mathrm{~ms}$ and $100 \mathrm{~ms}$ respectively, and were used to assess model efficacies, as described previously (McLean et al., 2003). There is increasing evidence to suggest that non-contact anterior cruciate ligament injuries occur within the first $50 \mathrm{~ms}$ of stance (Griffin et al., 2006). Hence, limiting validation of the predictive capacity of each model to the first $100 \mathrm{~ms}$ seems plausible.

\subsection{Extraction of knee joint loads}

For each optimized system, the external flexion-extension, varus-valgus and internal-external rotation knee reaction moment, and anterior tibial shear force were quantified at $1 \mathrm{~ms}$ intervals with respect to the tibial anatomical reference frame. Joint moment data were obtained directly from the dynamic equations of motion. Anterior shear was estimated by adding the resultant intersegmental force at the knee joint, similarly obtained from the equations of motion, to the combined optimized force contributions of the quadriceps and hamstring muscle groups (McLean et al., 2004a). The relative contribution of each muscle force was calculated using equations describing patellar and hamstring tendon orientations as a function of knee flexion angle (Herzog and Read, 1993). The knee joint coordinate system orientation was such that anterior shear force, the anterior component of the quadriceps force, and knee extension, valgus and external rotation loads applied to the joint were all defined as positive.

\subsection{Neuromuscular control effects on knee loading parameters}

Seven series of Monte Carlo simulation experiments were conducted to examine the effects of specific prescribed 
NMC changes on resultant peak anterior shear force $\left(\mathrm{FD}_{\text {Ant }}\right)$ and external knee valgus moment $\left(M_{\mathrm{Val}}\right)$ \&during the first $100 \mathrm{~ms}$ of sidestep stance. First, a baseline series $(\mathrm{n}=5000)$ of simulations was performed on each model, whereby random numbers were added to the initial measured body segment and angular positions and linear and angular velocities (McLean et al., 2003, 2004a). These numbers were generated for each model from a Gaussian distribution with zero mean and the standard deviation calculated in each movement variable across the ten sidestep cutting trials for the corresponding subject (McLean et al., 2003). Further, optimized activation parameters for the knee extensors (rectus femoris and vasti group) and knee flexors (hamstring group) were each multiplied by a separate Gaussian random number with a mean of one and a standard deviation of one (McLean et al., 2004a). The resulting muscle stimulation levels were limited to values between zero and one as per the model design.

The remaining six Monte Carlo simulation series' applied to each model represented sequential controlled experiments, corresponding to either a prescribed increase or decrease in one of three initial contact kinematic parameters. The three kinematic parameters, namely combined hip and knee flexion-extension position, hip internal-external rotation position, and hip internal-external rotation velocity, were chosen based on hypotheses arising from previous in vivo human experimentation (Chappell et al., 2007; Hewett et al., 2006a, 2006b; McLean et al., 2005b). Specifically, an isolated change (increase of decrease) was first prescribed to either initial hip and knee flexion-extension position $\left(5^{\circ}\right)$, hip internal-external rotation position $\left(5^{\circ}\right)$, or hip internal-external rotation velocity $\left(100^{\circ} . \mathrm{s}^{-1}\right)$ within each model. The magnitude of change in each of the three variables was based on mean inter-trial variations recorded previously for this same subject population (McLean et al., 2005b). The Monte Carlo simulation methods described above were then re-applied in each of the six cases, with resultant peak $\mathrm{FD}_{\mathrm{Ant}}$ and $M_{\mathrm{Val}}$ data again recorded over the 5000 simulations.

\subsection{Data analyses}

Peak stance $(0-200 \mathrm{~ms})$ phase values for $\mathrm{FD}_{\mathrm{Ant}}$, and opposing peaks for external knee flexion-extension $\left(M_{F E}\right)$, varus-valgus $\left(M_{\mathrm{VV}}\right)$ and internal-external rotation $\left(M_{\mathrm{IE}}\right)$ moments, obtained from each subject-based optimized simulation, were submitted to a one-way ANOVA to determine for the main effect of sex. A Bonferoni corrected alpha level of 0.007 was implemented to denote statistical significance. Effect size was also determined for each comparison according to Cohen (1988), where, by definition, large, medium and small effect sizes were defined by values greater than $0.8,0.5$ and 0.2 respectively. The number of ACL injuries via either an isolated anterior tibial shear or valgus load mechanism was quantified for each Monte Carlo Simulation series. Specifically, sagittal or transverse plane injuries were defined to occur when peak $F D_{\text {Ant }}$ or
$M_{\text {Val }}$ values exceeded $2000 \mathrm{~N}$ (Woo et al., 1991) or $125 \mathrm{Nm}$ (Seering et al., 1980) respectively. Non-parametric statistical methods (Friedman test) were then utilized to determine whether respective $(n=6)$ perturbations in each of the three initial contact kinematic parameters increased or decreased the number of ACL injuries compared to baseline Monte Carlo levels.

\section{Results}

The fit and prediction errors of the optimized subjectspecific movement simulations, over $200 \mathrm{~ms}$ and $100 \mathrm{~ms}$ respectively, were similar for male and female models (Table 1). For each of the 12 optimized model variables, the mean difference between measured and simulated data was less than two standard deviations. In fact, excluding the GRF data, mean differences were less than one standard deviation. All mean RMS model prediction errors were also below two standard deviations. The mean (SD) optimized muscle activation parameters $(n=5)$ for the rectus femoris, and vastus and hamstring muscle groups were also consistent both between individuals and across sex (Fig. 2).

Mean optimized external knee joint load patterns over the first $100 \mathrm{~ms}$ of stance were consistent between male and female-based models. Sex comparisons of peak joint loads within this time frame did not yield any statistically significant differences. Female models did however, present noticeably larger peak external $M_{\mathrm{Val}}$ values compared to male models, with a medium effect size being reported

\section{Table 1}

Mean (SD) validity measures for optimized model simulations of sidestepping as a function of gender. RMS fit error corresponds to the average difference in terms of SD's between simulated and measured data over the first $200 \mathrm{~ms}$ of stance. RMS prediction error is the ratio of the mean RMS difference between the ten sets (trials) of measured and simulated data, to the mean measured inter-trial variability over $100 \mathrm{~ms}$

\begin{tabular}{|c|c|c|c|c|}
\hline \multirow[t]{2}{*}{ Variable } & \multicolumn{2}{|c|}{ RMSFit/SD (200 ms) } & \multicolumn{2}{|c|}{ RMSPred/SD (100 ms) } \\
\hline & Male & Female & Male & Female \\
\hline $\begin{array}{l}\text { Medio-lateral force } \\
(\mathrm{Fx})\end{array}$ & $1.31(0.28)$ & $1.48(0.83)$ & $1.82(0.26)$ & $1.92(0.65)$ \\
\hline $\begin{array}{l}\text { Anterior-posterior } \\
\text { force (Fy) }\end{array}$ & $1.55(0.92)$ & $1.79(0.55)$ & $1.92(0.81)$ & $1.87(0.57)$ \\
\hline Vertical force $(\mathrm{Fz})$ & $1.44(0.38)$ & $1.62(0.76)$ & $1.69(0.36)$ & $1.72(0.83)$ \\
\hline Somersault (Rx) & $0.89(0.47)$ & $0.92(0.60)$ & $1.84(0.78)$ & $1.88(0.65)$ \\
\hline Tilt (Ry) & $0.89(0.68)$ & $0.98(0.58)$ & $1.77(0.55)$ & $1.64(0.71)$ \\
\hline Twist (Rz) & $0.54(0.40)$ & $0.73(0.49)$ & $1.02(0.34)$ & $1.13(0.60)$ \\
\hline $\begin{array}{l}\text { Hip flexion- } \\
\quad \text { extension }(\mathrm{Hx})\end{array}$ & $0.62(0.20)$ & $0.91(0.49)$ & $1.33(0.29)$ & $1.99(0.25)$ \\
\hline $\begin{array}{l}\text { Hip abduction- } \\
\text { adduction (Hy) }\end{array}$ & $0.53(0.21)$ & $0.56(0.49)$ & $1.47(0.62)$ & $1.09(0.32)$ \\
\hline $\begin{array}{l}\text { Hip axial rotation } \\
(\mathrm{Hz})\end{array}$ & $0.87(0.87)$ & $0.81(0.20)$ & $1.50(0.82)$ & $1.39(0.60)$ \\
\hline $\begin{array}{l}\text { Knee flexion-- } \\
\text { extension }(\mathrm{Kx})\end{array}$ & $0.63(0.28)$ & $0.63(0.42)$ & $1.34(0.75)$ & $1.34(0.87)$ \\
\hline $\begin{array}{l}\text { Ankle planter-dorsi } \\
\text { flexion }(\mathrm{Ax})\end{array}$ & $0.89(0.46)$ & $0.86(0.40)$ & $1.37(0.71)$ & $1.37(0.64)$ \\
\hline $\begin{array}{l}\text { Ankle pronation-- } \\
\text { supination (Ay) }\end{array}$ & $0.51(0.22)$ & $0.65(0.32)$ & $1.43(0.53)$ & $1.83(0.58)$ \\
\hline
\end{tabular}



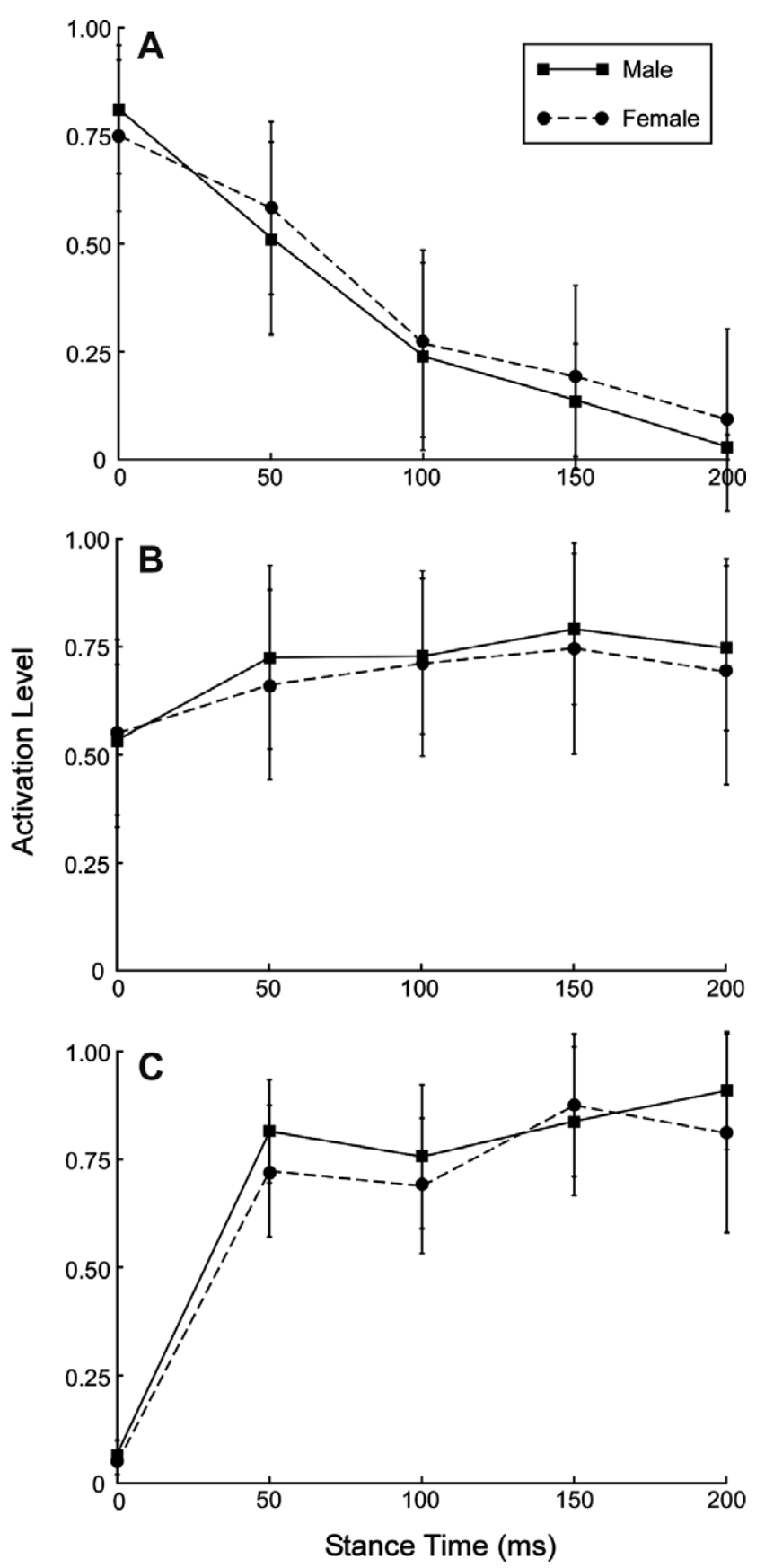

Fig. 2. Gender comparisons of mean $( \pm \mathrm{SD})$ hamstring $(\mathrm{A})$, rectis femoris (B) and vastus (C) muscle activation patterns obtained for the first $200 \mathrm{~ms}$ of sidestep stance in optimized model simulations.

(Table 2). Small to medium effect sizes were also calculated for sex comparisons of peak external $M_{\mathrm{Fle}}$ and $M_{I R}$, with female models demonstrating slightly lower values in each case compared to males over the first $100 \mathrm{~ms}$ of stance (see Table 2).

Baseline Monte Carlo simulations produced relatively large increases in peak $\mathrm{FD}_{\mathrm{Ant}}$ and $M_{\mathrm{Val}}$ measures in both male and female model simulations compared to optimized values (Fig. 3). Peak FD $_{\text {Ant }}$ measures however, did not come close to exceeding $2000 \mathrm{~N}$ in any perturbed model and hence no injuries were considered to occur via this
Table 2

Effect of gender on mean (SD) peak joint loads estimated during the stance phase $(0-200 \mathrm{~ms})$ of optimized sidestep cutting simulations

\begin{tabular}{llll}
\hline Dependent measure & Male & Female & Effect size $(r)$ \\
\hline FD $_{\mathrm{A}}(\mathrm{N})$ & $-523.9(568.0)$ & $-689.7(458.1)$ & 0.16 \\
$M_{\text {Ext }}(\mathrm{Nm})$ & $129.6(69.2)$ & $97.8(61.4)$ & 0.24 \\
$M_{\text {Fle }}(\mathrm{Nm})$ & $291.2(75.5)$ & $219.3(71.4)$ & 0.44 \\
$M_{\text {Val }}(\mathrm{Nm})$ & $64.0(18.8)$ & $85.3(17.2)$ & 0.51 \\
$M_{\text {Var }}(\mathrm{Nm})$ & $33.4(16.3)$ & $28.9(25.3)$ & 0.11 \\
$M_{\text {ER }}(\mathrm{Nm})$ & $26.5(20.9)$ & $36.6(17.2)$ & 0.26 \\
$M_{\text {IR }}(\mathrm{Nm})$ & $36.6(19.1)$ & $22.6(18.1)$ & 0.39 \\
\hline
\end{tabular}

mechanism. Conversely, $27.1(19.7) \%$ and $34.5(27.5) \%$ of baseline Monte Carlo simulations resulted in an injury via an isolated valgus loading mechanism for male and female models respectively.

Prescribed changes in the three initial contact kinematic parameters produced observable changes in ACL injury numbers via a valgus loading mechanism compared to baseline Monte Carlo values. Specifically, landing with either decreased combined hip and knee flexion, or decreased hip internal rotation velocity produced significantly $(P<0.05)$ more valgus load-induced ACL injuries (higher mean injury ranking) per 5000 perturbed simulations than baseline Monte Carlo levels (Fig. 4). Conversely, landing with increased hip and knee flexion, or increased hip internal rotation velocity resulted in significantly $(P<0.05)$ less (lower mean injury ranking) injuries compared to baseline values. Again, injuries via an isolated sagittal plane loading mechanism were not observed for any model, regardless of segment initial contact positions or velocities, and resultant perturbations.

\section{Discussion}

The current study examined the influence of specific neuromuscular control parameters on the resultant risk of non-contact ACL injury. Using a forward dynamics modeling approach, we also examined extreme knee loading states within a directed and completely controlled neuromuscular control framework, something that is unique to this methodological approach. Mean validation (RMS/ Fit) errors for both male and female models were well below our previously defined acceptance criteria of two standard deviations (McLean et al., 2003). Previous studies have adopted similar criteria for validating modeled movement simulations based on measured input conditions (Neptune et al., 2000). With regard to our own modeling, a range of two standard deviations is indicative of the between-trial variations observed in the lower limb kinematic profiles during sidestep movements (McLean et al., 1999, 2005b). For the current study, however, optimized models could simulate kinematic traces to well within one standard deviation. Hence, considering this fact and that kinematic traces and the associated quadriceps and hamstring muscle activation patterns were also consistent with those reported previously for sidestepping movements 

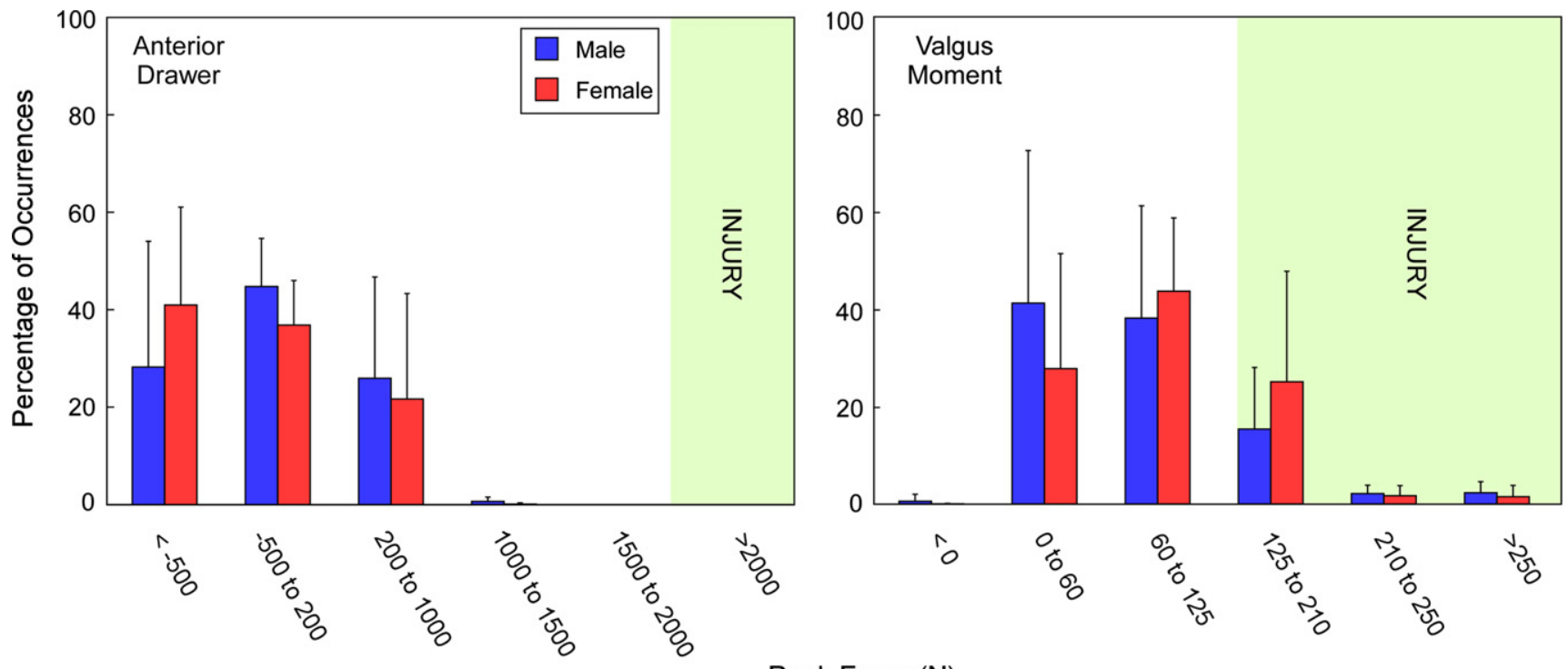

Peak Force (N)

Fig. 3. Effect of initial contact NMC perturbations $(n=5000)$ on mean resultant $(\mathrm{A})$ anterior drawer force $\left(\mathrm{FD}_{\mathrm{Ant}}\right)$ and $(\mathrm{B})$ valgus $\left(M_{\mathrm{Val}}\right)$ moments quantified in male and female sidestep models. ACL injury was deemed to occur when anterior drawer force and peak external valgus moment exceeded $2000 \mathrm{~N}$ (Woo et al., 1991) and $125 \mathrm{Nm}$ (Seering et al., 1980) respectively.

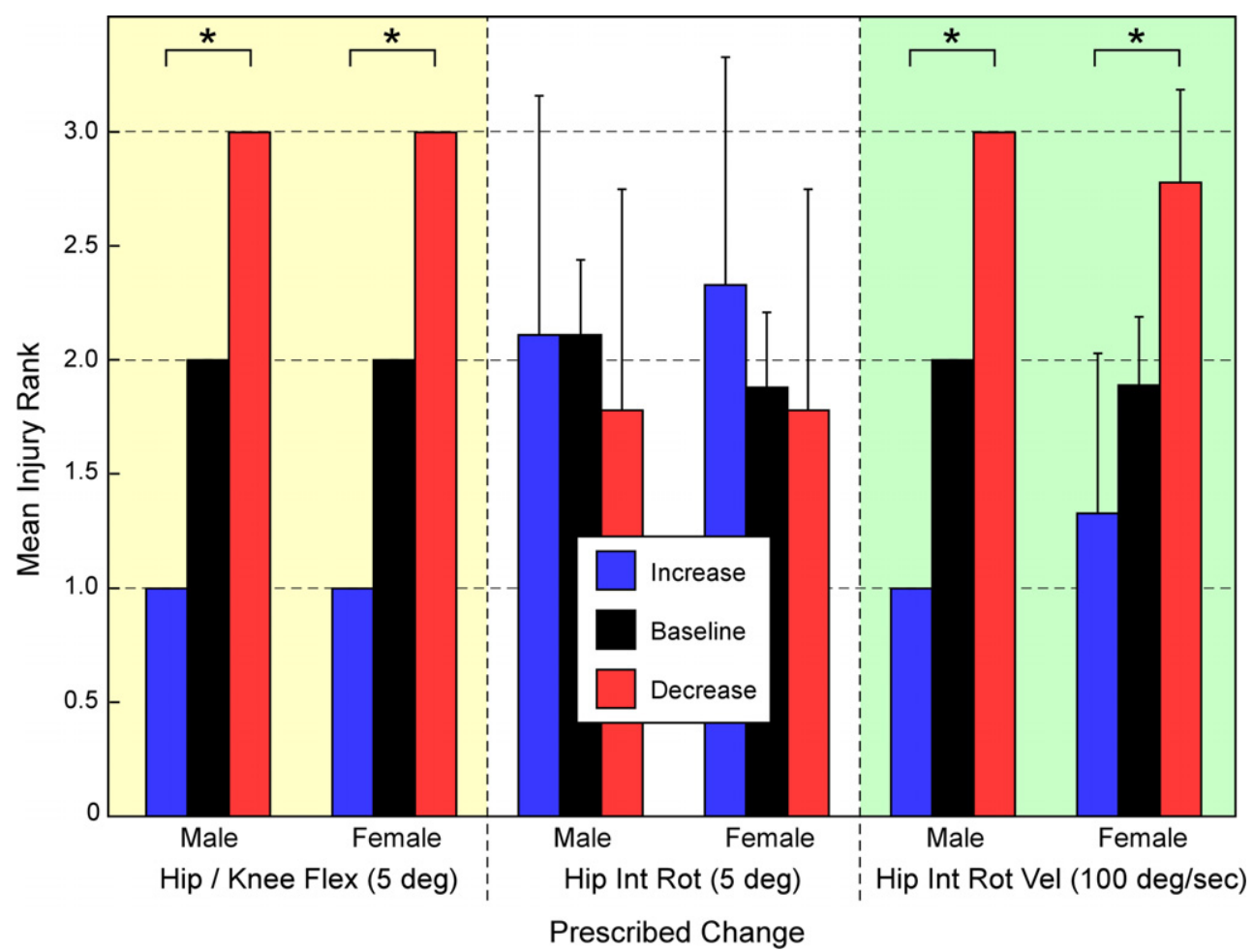

Fig. 4. Comparison of knee valgus load-induced mean ACL injury rankings for prescribed decreases and increases in initial contact hip and knee flexionextension posture, hip internal-external rotation posture and hip internal-external rotation velocity during simulated sidestep stance. Specifically, a higher mean injury rank corresponded directly to higher number of ACL injuries.

(Colby et al., 2000; McLean et al., 2005b), we felt that we could successfully simulate and test realistic sidestepping movements. Mean normalized RMS prediction errors, reflecting model abilities to reliably predict the consequences of perturbed neuromuscular control were also sub- stantially reduced compared to our previous sidestep models (McLean et al., 2003, 2004a). The adoption of fixed rather than optimized wobbling mass stiffness and damping parameters, as noted earlier, likely contributed to the improved predictive capacity of the current models. 
Mean optimized peak external knee joint magnitudes and their associated timings were consistent with those previously measured for sidestepping in this (McLean et al., 2005b) and other subject populations (McLean et al., 2007; Sigward and Powers, 2006), although subtle discrepancies in overall stance phase loading patterns were evident. Considering ACL integrity is ultimately governed by the magnitude of the externally applied load however, we are confident that these discrepancies did not compromise model abilities to predict injury risk. Optimized female models also had higher knee valgus loads than male models, reflecting sex-based differences typically observed experimentally in this parameter for jump landing (McLean et al., 2007) and sidestepping movements (McLean et al., 2005b; Sigward and Powers, 2006). This sex-disparity was again evident within baseline Monte Carlo measures, where female models produced far more valgus load-induced ACL injuries than did the male models. Knee valgus loading prospectively predicts ACL injury risk in young female athletes (Hewett et al., 2005)' precipitates extreme ACL load states in vitro (Kanamori et al., 2000; Markolf et al, 1995; Seering et al., 1980) and is the 3D knee load variable most sensitive to neuromuscular variability during sidestepping (McLean et al., 2003, 2004a). It appears plausible therefore, that the sex disparity in noncontact ACL injury rates may to some extent be explained by concomitant discrepancies in knee valgus loading elicited during dynamic landing postures such as sidestepping.

Baseline Monte Carlo data yielded valgus-induced ACL injury rates that seemed somewhat excessive in both male and female models. The Monte Carlo approach adopted within the current study necessarily considered each perturbed input parameter as independent from the next. In other words, for that $\mathrm{N}$-dimensional space, we necessarily sampled input conditions from all corners of the hypercube. Adopting such an approach meant that some of the combined perturbed conditions would be highly unlikely in vivo, with performance of a successful sidestep being virtually impossible in these instances. While this method necessarily exaggerates injury numbers compared to realworld situations, however, it does enable us to produce a model demonstrating enough injuries to successfully experiment with specific and realistic prevention parameters. Several secondary factors may have also contributed to the exaggerated valgus-induced ACL injury rates predicted by the perturbed models. By currently modeling the knee joint as a hinge, all out-of-plane knee rotations were effectively transferred to the hip joint, leading to potential overestimation of out-of-plane knee joint loads. Adding extra rotational degrees of freedom at the knee joint would likely negate this concern, similarly reducing the number of estimated ACL injuries, and we intend to address this issue in our ongoing modeling efforts. Regardless, however, basing relative injury risk on an explicit comparison of actual injury numbers meant that this systematic overestimation in no way adversely impacted the ensuing statistical analyses and hence the outcomes of the study. It should also be acknowledged that valgus-induced injury thresholds were currently derived from a very small number of cadaveric specimens (Seering et al., 1980). It is thus plausible that our adopted failure loads were substantially less than those required to truly rupture a young healthy $\mathrm{ACL}$ in vivo. Currently, limited insight exists regarding isolated and/or combined in vivo $3 \mathrm{D}$ knee joint load states that are indicative of ACL injury. Future research examining relationships between ACL and 3D knee load states associated with dynamic high risk sports postures thus appears well warranted.

Neuromuscular control perturbations failed to produce anterior tibial shear forces considered large enough to alone rupture the ACL (Woo et al., 1991). We have made similar observations in our previous work, where insights into this phenomenon are presented in detail (McLean et al., 2003, 2004a). Briefly, the combined relationships between joint angle and maximum muscle force (Delp et al., 1990), joint anatomy and ligament alignment/orientation (Delp et al., 1990; Pandy, 1997), quadriceps and ground reaction forces evident during initial deceleration (Shin et al., 2007), and the ensuing moment balance between these parameters as stance progresses (McLean et al., 2004a), likely place an effective ceiling on peak sagittal plane loads during sidestepping tasks. Of course, we acknowledge sagittal plane load contributions to ACL injury may have been underestimated in the current model. Adopting a homogeneous ultimate failure load threshold to denote injury may be problematic for example, especially considering the female ACL potentially exhibits decreased linear stiffness and ultimate failure loads compared to the male ACL (Chandrashekar et al., 2005, 2006). A sagittal plane injury mechanism may thus still prevail in a comparatively weaker female ACL. Failure to incorporate joint structural contributions to resultant 3D joint and ligament load states may also lead to erroneous interpretations of injury risk via a sagittal loading mechanism. A large posterior tibial slope for example, viewed to occur more frequently in women (Brandon et al., 2006), will likely promote increases in anteriorly directed tibial loads, and further orient the ACL such that a greater portion of this load is transferred along the ligament ( $\mathrm{Li}$ et al., 2006; Petersen and Zantop, 2007). Expanding current models to incorporate an anatomically relevant knee joint (Pflum et al., 2004; Shelburne et al., 2004) capable of accommodating subject-based variations in knee anatomies and laxities would provide immediate insights into individual predispositions to ACL injury based on joint and tissue biomechanical vulnerabilities.

Current results suggest that the potential for a valgusinduced ACL injury during sidestepping is to some extent directly governed by specific components of the preplanned neuromuscular strategy. Landing in a more extended (hip and knee) posture, for example, precipitated substantial increases in the number of ACL injuries, while landing with increased combined hip and knee flexion reduced this likelihood. Previous studies purport that 
increased hip and particularly knee extension at landing likely increases ACL injury risk by promoting debilitative anterior tibial shear loads (Chappell et al., 2007; Yu et al., 2006). Current results however, suggest that while the sagittal plane landing posture may indeed be an important predictor of injury risk, its' primary manifestation may be through an extreme out-of-plane, rather than sagittal knee load state. We are unsure as to the precise means through which this manifestation may arise. It is possible however, that landing in this position places the dominant sagittal muscle groups at sub-optimal lengths to effectively stabilize the joint against the large out-of-plane external loads evident at initial contact (Lloyd and Besier, 2003; Zhang et al., 2001). Regardless, current outcomes suggest that training individuals to land with increased hip and particularly knee flexion during dynamic landing tasks, which is already purported in many current prevention modalities (Hewett et al., 1999), is well warranted and should remain a key focus.

Landing with decreased and increased hip internal rotation velocities resulted in respective increases and decreases in the number of ACL injuries via a valgus loading mechanism. Every subject tested demonstrated a small yet definite hip internal rotation velocity at initial contact. Hence, decreasing this baseline parameter by $100 \%$ sec necessarily precipitated comparatively large initial contact external hip rotation velocities in the modeled sidestep maneuvers. The net impact of this modification was that an equally large hip external rotation posture was evident throughout stance in the perturbed sidestep. Peak stance phase knee valgus load during sidestepping has previously been linked to increased hip internal and not external rotation measures at landing (McLean et al., 2005b), rendering current outcomes somewhat counterintuitive. Prior observations however, were based on the in vivo assessment of normal sidestepping maneuvers that did not promote injurious joint loads. An internally rotated hip position at initial contact may thus represent a safe adaptive, rather than causative landing strategy. It is of course impossible to currently confirm or refute this assertion, and we remain unsure as to the exact means through which changes in initial contact hip rotation can influence ACL injury risk. Regardless, peak knee valgus load during sidestepping, and the subsequent potential for non-contact ACL injury appears particularly sensitive to initial contact hip transverse plane rotational velocities and should necessarily be considered within future prevention developments.

We currently examined the potential for three specific neuromuscular parameters to contribute to non-contact ACL injury. The utility of a forward dynamics modeling approach however, is such that any control parameter or combination of parameters can be altered, with the ensuing load states and the subsequent potential for injury readily evaluated. Hence, as experimental studies continue to present with further specific tenets linking neuromuscular control and ACL injury, methods such as that currently presented will become increasingly applicable and effective
(Erdemir et al., 2007). In saying this, however, we acknowledge that our current model is not without limitation. We have already commented for example, on the potential benefits of increased kinematic and structural complexity within the modeled knee joint. Furthermore, injury potential was currently based on the impact of isolated loads only. Dynamic landing postures such as sidestepping however, elicit complex combined 3D knee joint load states (McLean et al., 2007; Sigward and Powers, 2006), suggesting injury likely results through their combined impact. A more detailed understanding of the relationship between 3D knee joint and resultant ligament loading thus appears crucial.

There is no question that an integrative assessment of realistic neuromuscular, structural and mechanical contributions to non-contact ACL injury risk is extremely challenging. Considering the underlying injury mechanism likely presents through a combination of these factors however (Griffin et al., 2006), its revelation may only truly be possible through such an ambitious means. The development and continued evolution of computer based models such as that currently presented will ultimately provide an avenue though which ACL injury risk can be evaluated for a variety of dynamic landings at a mutlifactorial level, and with temporal efficiency. Without such a detailed and intricate understanding of the injury mechanism, prevention strategies will likely remain substantially compromised.

\section{Conclusions}

Using validated subject-specific forward dynamics models of sidestep cutting maneuvers, the current study demonstrated that variations in lower limb neuromuscular control precipitate maneuvers that are capable of injuring the ACL via an external valgus load mechanism. Furthermore, increases in the combined initial contact hip and knee flexion position or in the hip internal rotation velocity during these maneuvers decreases the potential for a valgusinduced ACL injury, with decreases in these same parameters having the opposite result. These control parameters should thus necessarily be considered for inclusion within evolving neuromuscular training modalities aimed at preventing non-contact ACL injuries. Ultimately however, successful prevention of these injuries only appears possible if the multifactorial nature of the underlying injury mechanism, incorporating neuromuscular and joint and ligament mechanical contributions, is considered and subsequently addressed.

\section{Conflict of interest statement}

No authors listed in conjunction with this manuscript submission.

\section{Acknowledgement}

This work was supported by U.S. National Institutes of Health (Grant 1 R01 AR47039). 


\section{References}

Agel, J., Arendt, E.A., Bershadsky, B., 2005. Anterior cruciate ligament injury in national collegiate athletic association basketball and soccer: a 13-year review. Am. J. Sports Med. 33, 524-530.

Bahr, R., Krosshaug, T., 2005. Understanding injury mechanisms: a key component of preventing injuries in sport. Brit. J. Sports Med. 39, 324-329.

Bell, A.L., Pedersen, D.R., Brand, R.A., 1990. A comparison of the accuracy of several hip center location prediction methods. J. Biomech. 23, 617-621.

Brandon, M.L., Haynes, P.T., Bonamo, J.R., Flynn, M.I., Barrett, G.R., Sherman, M.F., 2006. The association between posterior-inferior tibial slope and anterior cruciate ligament insufficiency. Arthroscopy 22, 894-899.

Chandrashekar, N., Slauterbeck, J., Hashemi, J., 2005. Sex-based differences in the anthropometric characteristics of the anterior cruciate ligament and its relation to intercondylar notch geometry: a cadaveric study. Am. J. Sports Med. 33, 1492-1498.

Chandrashekar, N., Mansouri, H., Slauterbeck, J., Hashemi, J., 2006. Sexbased differences in the tensile properties of the human anterior cruciate ligament. J. Biomech. 39, 2943-2950.

Chappell, J.D., Creighton, R.A., Giuliani, C., Yu, B., Garrett, W.E., 2007. Kinematics and electromyography of landing preparation in vertical stop-jump: risks for noncontact anterior cruciate ligament injury. Am. J. Sports Med. 35, 235-241.

Cohen, J., 1988. Statistical Power Analysis for the Behavioral Sciences, second ed. Hillsdale, New Jersey.

Colby, S., Francisco, A., Yu, B., Kirkendall, D., Finch, M., Garrett Jr., W., 2000. Electromyographic and kinematic analysis of cutting maneuvers. Implications for anterior cruciate ligament injury. Am. J. Sports Med. 28, 234-240.

de Leva, P., 1996. Adjustments to Zatsiorsky-Seluyanov's segment inertia parameters. J. Biomech. 29, 1223-1230.

Delp, S.L., Loan, J.P., Hoy, M.G., Zajac, F.E., Topp, E.L., Rosen, J.M., 1990. An interactive graphics-based model of the lower extremity to study orthopaedic surgical procedures. IEEE Trans. Biomed. Eng. 37, 757-767.

Erdemir, A., McLean, S., Herzog, W., van den Bogert, A.J., 2007. Modelbased estimation of muscle forces exerted during movements. Clin. Biomech. 22, 131-154.

Goffe, W.L., Ferrier, G.D., Rogers, J., 1994. Global optimization of statistical functions with simulated annealing. J. Econometr. 60, 6599.

Griffin, L.Y., Albohm, M.J., Arendt, E.A., Bahr, R., Beynnon, B.D., Demaio, M., Dick, R.W., Engebretsen, L., Garrett Jr., W.E., Hannafin, J.A., Hewett, T.E., Huston, L.J., Ireland, M.L., Johnson, R.J., Lephart, S., Mandelbaum, B.R., Mann, B.J., Marks, P.H., Marshall, S.W., Myklebust, G., Noyes, F.R., Powers, C., Shields Jr., C., Shultz, S.J., Silvers, H., Slauterbeck, J., Taylor, D.C., Teitz, C.C., Wojtys, E.M., Yu, B., 2006. Understanding and preventing noncontact anterior cruciate ligament injuries: a review of the Hunt Valley II meeting, January 2005. Am. J. Sports Med. 34, 1512-1532.

Herzog, W., Read, L.J., 1993. Lines of action and moment arms of the major force-carrying structures crossing the human knee joint. J. Anat. $182,213-230$

Hewett, T.E., Lindenfeld, T.N., Riccobene, J.V., Noyes, F.R., 1999. The effect of neuromuscular training on the incidence of knee injury in female athletes. A prospective study. Am. J. Sports Med. 27, 699-706.

Hewett, T.E., Myer, G.D., Ford, K.R., Heidt Jr., R.S., Colosimo, A.J., McLean, S.G., van den Bogert, A.J., Paterno, M.V., Succop, P., 2005. Biomechanical measures of neuromuscular control and valgus loading of the knee predict anterior cruciate ligament injury risk in female athletes: a prospective study. Am. J. Sports Med. 33, 492-501.

Hewett, T.E., Myer, G.D., Ford, K.R., 2006a. Anterior cruciate ligament injuries in female athletes: Part 1, mechanisms and risk factors. Am. J. Sports Med. 34, 299-311.
Hewett, T.E., Ford, K.R., Myer, G.D., Wanstrath, K., Scheper, M., 2006b. Gender differences in hip adduction motion and torque during a single-leg agility maneuver. J. Orthop. Res. 24, 416-421.

Isman, R.E., Inman, V.T., 1969. Anthropometric studies of the human foot and ankle. Bull. Prosth. Res. 11, 97-129.

Kanamori, A., Woo, S.L., Ma, C.B., Zeminski, J., Rudy, T.W., Li, G., Livesay, G.A., 2000. The forces in the anterior cruciate ligament and knee kinematics during a simulated pivot shift test: a human cadaveric study using robotic technology. Arthroscopy 16, 633-639.

Li, G., Papannagari, R., DeFrate, L.E., Yoo, J.D., Park, S.E., Gill, T.J., 2006. Comparison of the ACL and ACL graft forces before and after ACL reconstruction: an in vitro robotic investigation. Acta Orthop. 77, 267-274.

Lloyd, D.G., Besier, T.F., 2003. An EMG-driven musculoskeletal model to estimate muscle forces and knee joint moments in vivo. J. Biomech. 36, 765-776.

Lohmander, L.S., Ostenberg, A., Englund, M., Roos, H., 2004. High prevalence of knee osteoarthritis, pain, and functional limitations in female soccer players twelve years after anterior cruciate ligament injury. Arthritis Rheum. 50, 3145-3152.

Maletius, W., Messner, K., 1999. Eighteen- to twenty-four-year follow-up after complete rupture of the anterior cruciate ligament. Am. J. Sports Med. 27, 711-717.

Mandelbaum, B.R., Silvers, H.J., Watanabe, D.S., Knarr, J.F., Thomas, S.D., Griffin, L.Y., Kirkendall, D.T., Garrett Jr., W., 2005. Effectiveness of a neuromuscular and proprioceptive training program in preventing anterior cruciate ligament injuries in female athletes: 2-year follow-up. Am. J. Sports Med. 33, 1003-1010.

Markolf, K.L., Burchfield, D.M., Shapiro, M.M., Shepard, M.F., Finerman, G.A., Slauterbeck, J.L., 1995. Combined knee loading states that generate high anterior cruciate ligament forces. J. Orthop. Res. 13, 930-935.

McLean, S.G., Neal, R.J., Myers, P.T., Walters, M.R., 1999. Knee joint kinematics during the sidestep cutting maneuver: potential for injury in women. Med. Sci. Sports Exerc. 31, 959-968.

McLean, S.G., Su, A., van den Bogert, A.J., 2003. Development and validation of a 3-D model to predict knee joint loading during dynamic movement. J. Biomech. Eng. 125, 864-874.

McLean, S.G., Huang, X., Su, A., Van Den Bogert, A.J., 2004a. Sagittal plane biomechanics cannot injure the ACL during sidestep cutting. Clin. Biomech. 19, 828-838.

McLean, S.G., Lipfert, S.W., Van Den Bogert, A.J., 2004b. Effect of gender and defensive opponent on the biomechanics of sidestep cutting. Med. Sci. Sports Exerc. 36, 1008-1016.

McLean, S.G., Huang, X., van den Bogert, A.J., 2005a. Association between lower extremity posture at contact and peak knee valgus moment during sidestepping: implications for ACL injury. Clin. Biomech. 20, 863-870.

McLean, S.G., Walker, K.B., van den Bogert, A.J., 2005b. Effect of gender on lower extremity kinematics during rapid direction changes: an integrated analysis of three sports movements. J. Sci. Med. Sport 8, $411-422$.

McLean, S.G., Felin, R.E., Suedekum, N., Calabrese, G., Passerallo, A., Joy, S., 2007. Impact of fatigue on gender-based high-risk landing strategies. Med. Sci. Sports. Exerc. 39, 502-514.

Neptune, R.R., Wright, I.C., van Den Bogert, A.J., 2000. A method for numerical simulation of single limb ground contact events: application to heel-toe running. Comput. Methods Biomech. Biomed. Eng. 3, 321-334.

Pandy, M.G.a.S., K.B., 1997. Dependence of cruciate-ligament loading on muscle forces and external load. J. Biomech. 30, 1015-1024.

Petersen, W., Zantop, T., 2007. Anatomy of the anterior cruciate ligament with regard to its two bundles. Clin. Orthop. Relat. Res. 454, 3547.

Pflum, M.A., Shelburne, K.B., Torry, M.R., Decker, M.J., Pandy, M.G., 2004. Model prediction of anterior cruciate ligament force during drop-landings. Med. Sci. Sports. Exerc. 36, 1949-1958. 
Pollard, C.D., Sigward, S.M., Powers, C.M., 2007. Gender differences in hip joint kinematics and kinetics during side-step cutting maneuver. Clin. J. Sport. Med. 17, 38-42.

Seering, W.P., Piziali, R.L., Nagel, D.A., Schurman, D.J., 1980. The function of the primary ligaments of the knee in varus-valgus and axial rotation. J. Biomech. 13, 785-794.

Shelburne, K.B., Pandy, M.G., Anderson, F.C., Torry, M.R., 2004. Pattern of anterior cruciate ligament force in normal walking. J. Biomech. 37, 797-805.

Shin, C.S., Chaudhari, A.M., Andriacchi, T.P., 2007. The influence of deceleration forces on ACL strain during single-leg landing: a simulation study. J. Biomech. 40, 1145-1152.

Sigward, S.M., Powers, C.M., 2006. The influence of gender on knee kinematics, kinetics and muscle activation patterns during side-step cutting. Clin. Biomech. 21, 41-48.

van den Bogert, A.J., Smith, G.D., Nigg, B.M., 1994. In vivo determination of the anatomical axes of the ankle joint complex: an optimization approach. J. Biomech. 27, 1477-1488.

Vaughan, C.L., Davis, B.L., O’Connor, J.C., 1990. Dynamics of Human Gait. Human Kinetics Publishers, Champaign, IL.
Wakeling, J.M., Nigg, B.M., 2001. Soft-tissue vibrations in the quadriceps measured with skin mounted transducers. J. Biomech. 34, 539-543.

Woo, S.L., Hollis, J.M., Adams, D.J., Lyon, R.M., Takai, S., 1991. Tensile properties of the human femur-anterior cruciate ligament-tibia complex. The effects of specimen age and orientation. Am. J. Sports Med. 19, 217-225.

Wu, G., Siegler, S., Allard, P., Kirtley, C., Leardini, A., Rosenbaum, D., Whittle, M., D'Lima, D.D., Cristofolini, L., Witte, H., Schmid, O., Stokes, I., 2002. ISB recommendation on definitions of joint coordinate system of various joints for the reporting of human joint motion part I: ankle, hip, and spine. International Society of Biomechanics. J. Biomech. 35, 543-548.

Yeadon, M.R., 1990. The simulation of aerial movement - I. The determination of orientation angles from film data. J. Biomech. 23, 5966.

Yu, B., Lin, C.F., Garrett, W.E., 2006. Lower extremity biomechanics during the landing of a stop-jump task. Clin. Biomech. 21, 297-305.

Zhang, L.Q., Xu, D., Wang, G., Hendrix, R.W., 2001. Muscle strength in knee varus and valgus. Med. Sci. Sports. Exerc. 33, 1194-1199. 